\title{
Orientation-dependent toxic effect of human papillomavirus type 33 long control region DNA in Escherichia coli cells
}

\author{
Eszter Gyöngyösi ${ }^{1}$ · Anita Szalmás ${ }^{1}$ · József Kónya ${ }^{1}$ · György Veress ${ }^{1}$ (i)
}

Received: 21 January 2020 / Accepted: 20 March 2020 / Published online: 3 April 2020

(c) The Author(s) 2020

\begin{abstract}
The functional analysis of human papillomavirus (HPV) sequence variation requires the molecular cloning of different genomic regions of virus variants. In this study, we report an unexpected difficulty experienced when trying to clone HPV33 long control region (LCR) variants in Escherichia coli. Standard cloning strategies proved to be inappropriate to clone HPV33 LCR variants in the forward orientation into a eukaryotic reporter vector (pGL2-Basic). However, by slight modification of culture conditions (incubation at $25^{\circ} \mathrm{C}$ instead of $37^{\circ} \mathrm{C}$ ), constructs containing the HPV33 LCR variants in the forward orientation were obtained. Transformation experiments performed with different HPV33 LCR constructs indicated that there is a sequence element in the 5' LCR of HPV33 causing temperature-dependent toxic effect in E. coli. Sequence analysis revealed the presence of an open reading frame (ORF) in the 5' part of HPV33 LCR potentially encoding a 116amino acid polypeptide. Protein structure prediction suggested that this putative protein might have a structural similarity to transmembrane proteins. Even a low-level expression of this protein may cause significant toxicity in the host bacteria. In silico analysis of the LCR of HPV33 and some other HPV types belonging to the species Alphapapillomavirus 9 (HPV31, 35 and 58) seemed to support the assumption that the ORFs found in the 5' LCR of these HPVs are protein-coding sequences. Further studies should be performed to prove that these putative proteins are really expressed in the infected host cells and to identify their function.
\end{abstract}

Keywords Human papillomavirus $33 \cdot$ Long control region $\cdot$ Cloning $\cdot$ Toxicity

\section{Introduction}

Genital human papillomaviruses (HPV) belong to the Alpha genus of the Papillomaviridae family [1]. Within the Alphapapillomavirus genus, the high-risk or oncogenic HPV types (such as HPV16, 18, 31, and 33) are causally associated with the development of premalignant and malignant lesions of the uterine cervix [2]. In certain high-risk HPV types, intra-type sequence variation was shown to be related with

Edited by William Dundon.

Electronic supplementary material The online version of this article (https://doi.org/10.1007/s11262-020-01754-4) contains supplementary material, which is available to authorised users.

György Veress

veregy@med.unideb.hu

1 Department of Medical Microbiology, Faculty of Medicine, University of Debrecen, Nagyerdei krt. 98, 4032 Debrecen, Hungary variable oncogenic potential [3]. In an effort to explain the differences in the clinical behaviour of intra-type sequence variants, in vitro functional studies have been performed targeting different genomic regions of the HPV variants [4]. The long control region (LCR) of HPV variants has been the subject of several functional studies [5-10].

The LCR of HPVs regulates the replication and gene expression of the virus [11]. It contains binding sites for viral and cellular transcription factors. In the Alphapapillomaviruses, there are four binding sites for the viral E2 proteins in a conserved arrangement [11]. The LCR is composed of three functional parts (Fig. 1). The $5^{\prime}$ LCR contains a polyadenylation site for the late viral transcripts. The central LCR contains the epithelial-specific enhancer, while the $3^{\prime}$ LCR contains the origin of replication and the promoter for the early viral genes E6 and E7. The LCR of papillomaviruses is not known to encode any viral proteins.

The functional analysis of HPV sequence variation requires the molecular cloning of different genomic regions of virus variants. This is usually a routine task, but in certain 


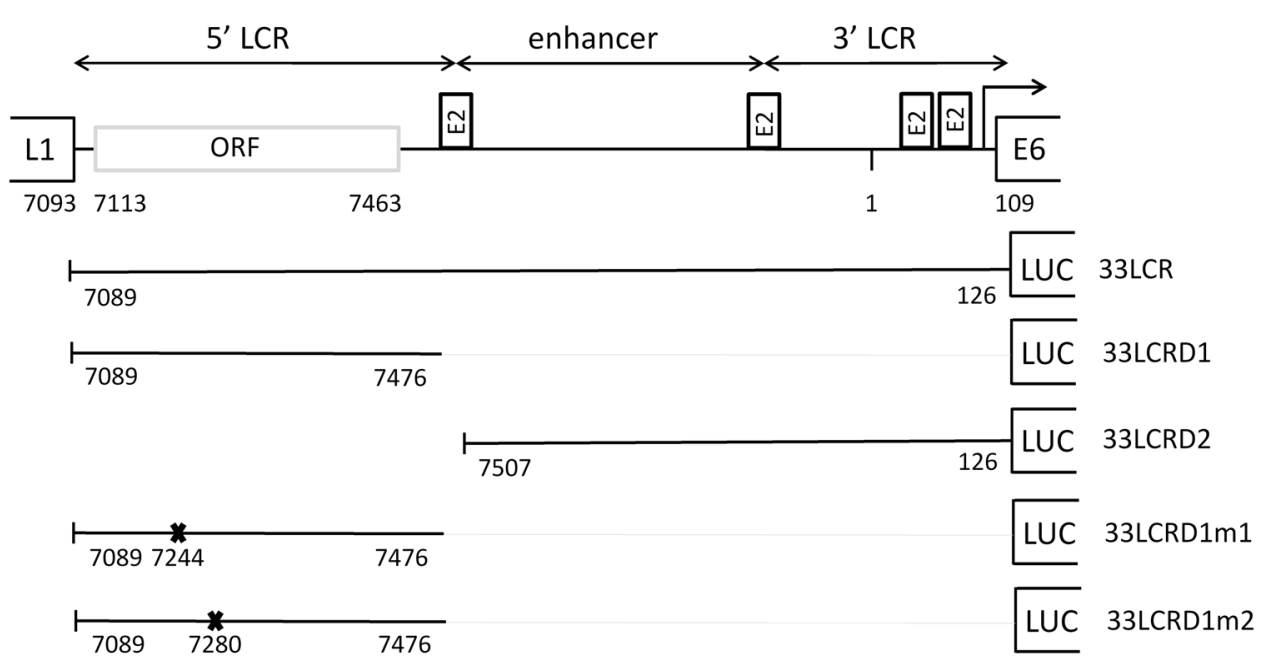

Fig. 1 Schematic representation of the different HPV33 LCR constructs used in this study. The locations of the four E2 binding sites and the three functional segments of HPV33 LCR are shown above. A previously unreported ORF found in the $5^{\prime}$ LCR segment is also shown. Numbers indicate nt 1 of the circular genome and the nt posi-

HPV types, viral regions or sequence variants may occur that are hard to clone using established methods. Here, we report an unexpected difficulty experienced when trying to clone HPV33 LCR variants in E. coli. We show that the HPV33 LCR has an orientation-dependent toxic effect in certain $E$. coli strains. This effect can be localised to the $5^{\prime}$ LCR and might be caused by an open reading frame (ORF) potentially encoding a 116-amino acid polypeptide.

\section{Materials and methods}

\section{Sample collection, virus isolation, and genotyping}

Exfoliated cell samples were collected from the cervix of women with cytological and/or colposcopical abnormalities at the Department of Obstetrics and Gynaecology, University of Debrecen (Debrecen, Hungary). The isolation of viral DNA from the samples was performed with the High Pure Viral Nucleic Acid Kit (Roche, Basel, Switzerland) according to the protocol of the manufacturer. HPV genotyping was performed using the GenoFlow HPV Array Test kit (DiagCor, Kowloon Bay, Hong Kong). Samples found to be positive for HPV33 DNA were selected for detailed sequence analysis of the LCR.

\section{Polymerase chain reaction (PCR) and cloning}

To amplify the whole LCR region of HPV33 variants, the primers 33LCR129 and 33LCR1075 (containing restriction enzyme recognition sites for KpnI and HindIII enzymes, tions of the ORFs (shown as rectangles). The reporter constructs shown contain either the full-length HPV33 LCR or different fragments (33LCRD1 and 33LCRD2) or mutants (33LCRD1m1 and 33LCRD1m2) of the LCR each cloned in the forward orientation into the luciferase reporter vector pGL2-Basic

respectively) were used as shown in Supplementary material 1. PCR reactions were performed using the GeneAmp High Fidelity PCR System (Applied Biosystems, Foster City, CA, USA) according to the manufacturer's recommendations. In $50 \mu \mathrm{l}$ final reaction volume, $0.1-0.2 \mu \mathrm{g}$ sample DNA, $5 \mu$ GeneAmp High Fidelity 10X PCR Buffer with $\mathrm{MgCl}_{2}, 500 \mathrm{nM}$ of each primer, $200 \mathrm{nM}$ dNTPs and 2.5 Units GeneAmp High Fidelity Enzyme Mix were included. Amplification was performed on GeneAmp PCR System 2700 (Applied Biosystems). The amplification profile was as follows: $94{ }^{\circ} \mathrm{C}$ for $2 \mathrm{~min}$, then 35 cycles at $94{ }^{\circ} \mathrm{C}$ for $30 \mathrm{~s}, 55^{\circ} \mathrm{C}$ for $30 \mathrm{~s}$ and $72{ }^{\circ} \mathrm{C}$ for $60 \mathrm{~s}$. The $72{ }^{\circ} \mathrm{C}$ step was increased by $5 \mathrm{~s}$ per cycle in the last 25 cycles. After agarose gel electrophoresis, the PCR amplimers were cut from the gels and purified using Qiaquick Gel Extraction Kit (Qiagen, Hilden, Germany). Sequencing of purified PCR products was carried out by UD-GenoMed Medical Genomic Technologies Ltd. (Debrecen, Hungary) using the PCR primers and the Big Dye Terminator v3.1 Cycle Sequencing Kit (Applied Biosystems) and ABI Prism 3100-Avant Genetic Analyzer (Applied Biosystems).

In the first cloning approach, molecular cloning of a representative sequence variant of the HPV33 LCR into the promoterless luciferase reporter vector pGL2-Basic (Promega, Madison, WI, USA) was attempted in an orientation-directed manner. To this end, PCR products, obtained from the LCR of HPV33 and the pGL2-Basic vector were both digested with KpnI and HindIII restriction enzymes and purified with Qiaquick PCR Purification Kit (Qiagen). Ligation was performed with T4 DNA Ligase (New England Biolabs, Ipswich, MA, USA) at 
room temperature for $2 \mathrm{~h}$. The TransformAid Bacterial Transformation Kit (Thermo Fischer Scientific, Waltham, MA, USA) was used to transform ligation products into XL1, XL1-Blue or DH5 $\alpha$ E. coli strains according to the protocol of the manufacturer. Culturing of $E$. coli bacteria was performed on Luria Bertani (LB) agar containing $100 \mu \mathrm{g} / \mathrm{ml}$ ampicillin at $37{ }^{\circ} \mathrm{C}$ for about $16 \mathrm{~h}$ or at $25^{\circ} \mathrm{C}$ for about $48 \mathrm{~h}$.

In the second approach (TA cloning), the TOPO TA Cloning Kit with One Shot TOP10 chemically competent $E$. coli cells (Invitrogen, Carlsbad, CA, USA) was used to clone the selected full-length HPV33 LCR variants into a cloning vector (PCR 2.1-TOPO vector). Here, we used similar primers (33LCR129/2 and 33LCR1075/2) and PCR conditions as in the first approach, but the primers contained no restriction enzyme recognition sites. After the PCR reaction, A-addition was performed with Taq DNA Polymerase (Sigma, Saint Louis, MO, USA), then the cloning reaction and transformation (selection with kanamycin at $37{ }^{\circ} \mathrm{C}$ or $25^{\circ} \mathrm{C}$ ) was performed as described above.

To check the orientation of the inserts in the constructs obtained by the TA cloning method, colony PCR was used with primers annealing to the insert (HPV33LCR129/2 or HPV33LCR1075/2) and the cloning plasmid (M13 Forward primer) using REDTaq ReadyMix PCR Reaction Mix (Sigma). The amplification profile used was as follows: $94{ }^{\circ} \mathrm{C}$ for $10 \mathrm{~min}$, then 30 cycles at $94{ }^{\circ} \mathrm{C}$ for $30 \mathrm{~s}, 55^{\circ} \mathrm{C}$ for $1 \mathrm{~min}$ and $72^{\circ} \mathrm{C}$ for $1 \mathrm{~min} 30 \mathrm{~s}$. In the PCR with the primer pair M13 Forward and HPV33LCR129/2, amplimers of around 1,000 bp indicate clones containing the HPV33 LCR in the forward orientation. In the PCR with the primer combination M13 Forward and HPV33LCR1075/2, amplimers of the same length indicate clones containing the insert in the reverse orientation.

To prepare constructs containing deletions in the HPV33 LCR, the same PCR conditions and cloning methods were used as described for directional cloning, except for the primer pairs. To prepare the construct pGL2B-33LCRD1, the primers 33LCR129 and 33LCR516 were used. To prepare the construct pGL2B-33LCRD2, the primer pair 33LCR547-33LCR1075 was used. The template used here was the construct pGL2B-33LCR containing the full-length HPV33 prototype LCR.

Mutagenesis of the pGL2B-33LCRD1 construct was performed using the GeneArt site-directed mutagenesis system (Thermo Fischer Scientific, Waltham, MA, USA) using the primers shown in Supplementary material 1. In the pGL2B-33LCRD1m1 construct, the mutations result in a stop codon at amino acid no. 44 of the putative protein and a change of methionine to leucine at position 45 . In the pGL2B-33LCRD1 2 construct, the mutations result in a stop codon at codon 56 and a change of methionine to leucine at codon 57. A schematic representation of different constructs can be seen on Fig. 1. Each plasmid construct was verified by sequencing.

The number of bacterial colonies was counted by the OpenCFU software (https://opencfu.sourceforge.net/) [12].

\section{Bioinformatics tools}

The nucleotide sequences of HPV33 and related HPV types (belonging to the Alphapapillomavirus 9 species) were obtained from the PaVE (Papillomavirus Episteme) database (https://pave.niaid.nih.gov). In case of HPV31, the sequence was obtained from GenBank (https://www. ncbi.nlm.nih.gov/genbank, accession number HQ537666), because the HPV31 prototype sequence found in PaVE contains sequencing errors in the LCR [6]. The NCBI ORF finder site (https://www.ncbi.nlm.nih.gov/orffinder) was used to search for potential ORFs (open reading frames) in the LCR of the selected HPV types. We searched for ORFs of a minimal length of $150 \mathrm{nt}$ starting with ATG start codon. The LCRs of different HPVs were aligned using MACSE (multiple alignment of coding sequences) software [13]. The MLOGD (Maximum-Likelihood Overlapping Gene Detector) software (https://guinevere.otago.ac.nz/aef/MLOGD/ index.html) was used to estimate the probability that the ORFs found in the LCR of certain HPVs might have coding capacity $[14,15]$. Protein tertiary structure prediction was performed using the ab initio protein structure prediction tool QUARK (https://zhanglab.ccmb.med.umich.edu/ QUARK) [16, 17]. Protein sequence similarity searches were performed using Protein BLAST (https://blast.ncbi. nlm.nih.gov/Blast.cgi). Amino acid sequence alignment of the putative proteins potentially encoded by the LCR ORFs was obtained by the alignment of coding sequences (derived from ORF finder) performed by MACSE. The nucleotide and amino acid sequence alignments provided by MACSE were visualised by SeaView (https://doua.prabi.fr/software/ seaview) [18].

\section{Results}

\section{Cloning of HPV33 LCR may be hampered by the orientation-dependent toxic effect of viral DNA}

Most of the clinical samples that were found to be positive for HPV33 with the GenoFlow HPV Array Test kit could be amplified with the HPV33 LCR-specific primers producing amplimers of the expected size. However, an unexpected difficulty was met when trying to clone the PCR products into the promoterless luciferase reporter vector pGL2-Basic using established cloning techniques (including culturing the bacteria at $37^{\circ} \mathrm{C}$ ). After several trials with different HPV33 
LCR variants and different $E$. coli strains (XL1, XL1-Blue, and $\mathrm{DH} 5 \alpha$ ), only one clone was found that seemed to contain an insert with the appropriate length, but sequencing of the clone showed that it contained no HPV DNA.

Using the TA cloning approach, several clones were obtained that seemed to contain inserts of appropriate length. However, based on the results of orientation-specific PCR tests, all the clones obtained by this approach were found to contain the inserts in the reverse orientation (Fig. 2). This was confirmed by sequencing of selected clones and reproduced several times using different HPV33 LCR variants as PCR templates. This finding was quite unexpected as using TA cloning, one would expect to obtain constructs containing the insert with the forward and the reverse orientation at about the same frequency. If one of the two orientations is not obtained it may refer to the presence of a toxic element in the DNA sequence to be cloned. Therefore, we decided to modify the protocol to reduce the apparent toxicity of the LCR sequence by incubation of bacteria after transformation at $25{ }^{\circ} \mathrm{C}$ instead of $37^{\circ} \mathrm{C}$. This simple method was reported to be effective in the construction and propagation of infectious JEV (Japanese encephalitis virus) cDNA clones in $E$. coli [19].

This simple modification of the cloning protocol proved to be very effective also in this case. When bacteria were incubated at $25^{\circ} \mathrm{C}$ (for $48 \mathrm{~h}$ ) after transformation, a higher number of colonies was obtained compared to parallel plates incubated at $37{ }^{\circ} \mathrm{C}$ overnight. In addition, about half of the colonies obtained by incubation at $25^{\circ} \mathrm{C}$ proved to contain the cloned HPV33 LCR insert in the forward orientation (Supplementary material 2). The constructs obtained by this protocol were found to be stable even after further propagation in broth culture at $25^{\circ} \mathrm{C}$ (that was used for plasmid preparation). Sequencing of the constructs confirmed the proper (i.e. forward) orientation of the viral insert in the constructs and showed that no mutations occurred compared to the sequences of the original PCR products.

Next, the directional cloning protocol (cloning of viral insert into pGL2-Basic in the forward orientation) was also modified by incubation of bacteria at $25^{\circ} \mathrm{C}$ instead of $37{ }^{\circ} \mathrm{C}$ after the transformation step. This modification proved to be very effective also in this case as we were able to clone 6 different HPV33 LCR variants into the reporter vector in the proper (forward) orientation (shown in Supplementary material 3). The constructs were confirmed by sequencing and proved to be stable over time, similarly to the TA cloning protocol. These constructs were also used in luciferase reporter assays performed in human cell lines to study the transcriptional activities of the different HPV33 LCR variants (data not shown here).

\section{Confirmation and characterisation of the toxic effect of HPV33 LCR on host bacteria}

Next, transformation experiments were performed to confirm the temperature-dependent toxic effect of the cloned HPV33 LCR DNA on the host bacteria. To this end, XL1 bacteria were transformed by different amounts of a reporter construct containing the whole HPV33 prototype LCR cloned in the forward orientation into the luciferase reporter vector pGL2-Basic (pGL2B-33LCR). After transformation, the plates were incubated at $37{ }^{\circ} \mathrm{C}$ overnight or at $25^{\circ} \mathrm{C}$ for about $48 \mathrm{~h}$. As shown in Fig. 3, fewer colonies were formed at $37^{\circ} \mathrm{C}$ than at $25^{\circ} \mathrm{C}$ when plates containing bacteria transformed by the same amount of plasmid DNA were compared. The stability of the clones obtained at different temperatures was tested by sub-culturing on agar plates. Each of the clones formed at $25{ }^{\circ} \mathrm{C}$ were found to be stable when sub-cultured at $25^{\circ} \mathrm{C}$, while only a few clones formed at $37{ }^{\circ} \mathrm{C}$ could be sub-cultured at $37^{\circ} \mathrm{C}$. These results seem to confirm the toxicity of HPV33 LCR DNA at $37{ }^{\circ} \mathrm{C}$ when cloned in the forward orientation into the reporter vector pGL2-Basic.

To see if the toxicity of the HPV33 LCR prototype sequence is also retained in the natural sequence variants, reporter constructs containing 4 different HPV 33 LCR variants (shown in Supplementary Material 3) were tested in transformation experiments along with the empty reporter

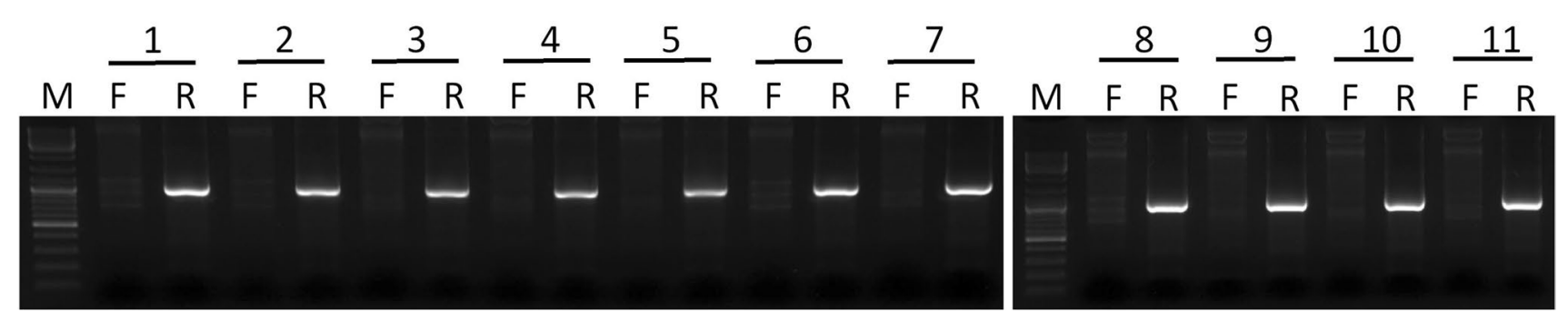

Fig. 2 Colony PCR analysis of clones obtained when trying to clone the HPV33 LCR region into the PCR 2.1-TOPO vector using the original protocol (cultivation of bacteria at $37{ }^{\circ} \mathrm{C}$ ). The different clones analysed by the PCR are indicated by different numbers.
F: PCR reaction specific for constructs containing the HPV33 LCR insert in the forward orientation. R: PCR reaction specific for constructs containing the HPV33 LCR insert in the reverse orientation 
Fig. 3 Results of transformation experiments performed with the construct pGL2B-33LCR containing the complete LCR of the HPV33 prototype. XL1 bacteria were transformed by either $5 \mathrm{ng}$ (a, d) or $0.5 \mathrm{ng}(\mathbf{b}, \mathbf{e})$ of the pGL2B-33LCR construct and incubated at $37^{\circ} \mathrm{C}$ overnight (a, b) or at $25^{\circ} \mathrm{C}$ for $48 \mathrm{~h}(\mathbf{d}, \mathbf{e})$. C: 12 colonies from the plate new agar plate and incubated at $37^{\circ} \mathrm{C}$ overnight. F: 12 colonies from the plate shown on $\mathrm{E}$ were streaked on a new agar plate and incubated at $25^{\circ} \mathrm{C}$ for $48 \mathrm{~h}$ shown on A were streaked on a
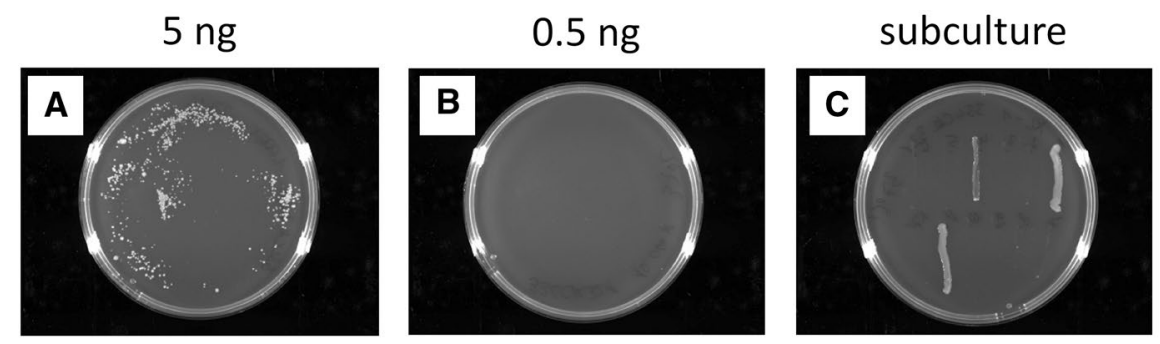

$37^{\circ} \mathrm{C}$
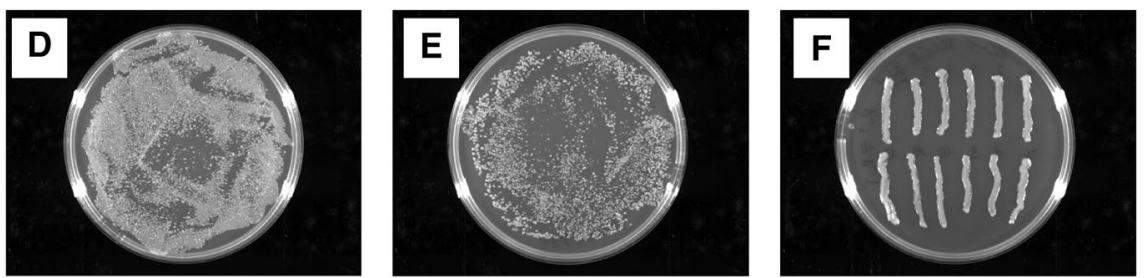

$25^{\circ} \mathrm{C}$

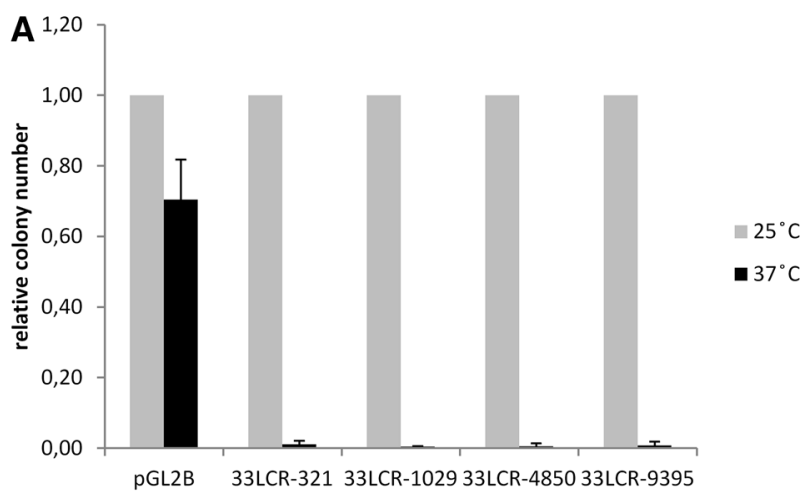

vector (pGL2-Basic). As shown in Fig. 4a, each of the constructs containing the different HPV33 LCR variants proved to be highly toxic at $37^{\circ} \mathrm{C}$.

In order to localise the region of HPV33 LCR that is responsible for the toxic effect on the bacterial host, transformation experiments were performed with deletion constructs containing different fragments of the HPV 33 prototype LCR (33LCRD1 and 33LCRD2) (Fig. 1). The results shown on Fig. 4b indicate that the toxic effect of HPV33 LCR can be localised to the region between nt 7089 and 7476 (represented by the 33LCRD1 construct). It is of note that the analysis of the HPV33 reference sequence (obtained from PaVE) performed by ORF finder revealed the presence of a putative ORF in the 5' part of HPV33 LCR (nt 7113-7463) potentially encoding a 116-amino acid protein (Fig. 1).

Next, two mutants were prepared from the pGL2B33LCRD1 construct in which the ORF present in the 5' LCR segment is disrupted by stop codons (Fig. 1). The 33LCRD1 1 construct showed reduced toxicity in transformation experiments compared to the parent construct (33LCRD1), while in the 33LCRD1m2 construct, the mutations disrupting the ORF resulted in a complete loss of toxicity of the LCR fragment (Fig. 4b). Taken together, the results of transformation experiments strongly suggest that the toxic effect of HPV33 LCR DNA on the host bacteria is caused by expression of a protein from the ORF found in the $5^{\prime} \mathrm{LCR}$.

\section{In silico analysis of the coding capacity of 5' LCR ORFs found in certain HPV types belonging to the species Alphapapillomavirus 9}

The ORF finder of NCBI revealed the presence of ORFs of more than $150 \mathrm{nt}$ length (with ATG start codons) in the 5' LCR of HPV33 and some other HPV types belonging to the species Alpha-9 (HPV31, 35 and 58) (Table 1.). No similar

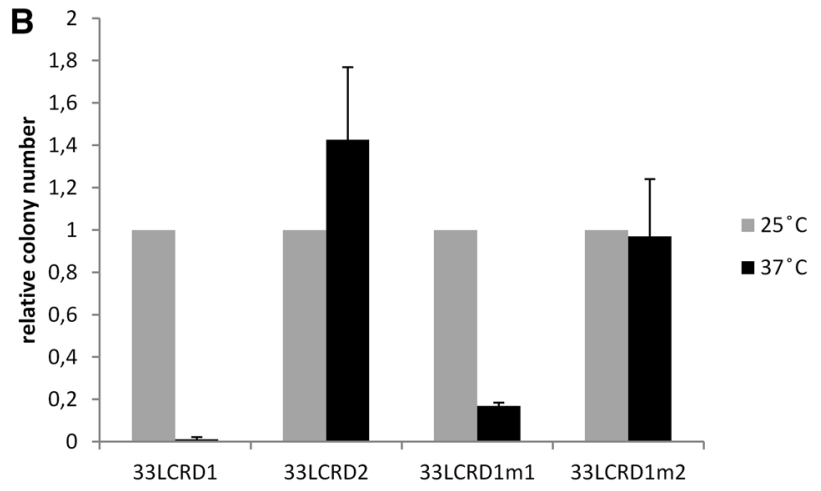

Fig. 4 Results of transformation experiments performed with different HPV33 LCR constructs. a Results of experiments performed with constructs containing the full-length LCR of different HPV33 variants (shown in Supplementary material 3) cloned into the vector pGL2-Basic. b Results of experiments performed with constructs containing different deletions or mutations as shown on Fig. 1. In each experiment, XL1 bacteria were transformed by $500 \mathrm{pg}$ of the different plasmid constructs and incubated at $37{ }^{\circ} \mathrm{C}$ overnight or at $25{ }^{\circ} \mathrm{C}$ for 48 as indicated. The number of colonies was counted by OpenCFU (https://opencfu.sourceforge.net/). For each construct, the colony number obtained at $25{ }^{\circ} \mathrm{C}$ was set to 1 and the colony number obtained at $37{ }^{\circ} \mathrm{C}$ is shown relative to this (standardised colony number). The means and standard deviations of 3 independent experiments are shown 
Table 1 Positions and characteristics of ORFs found in the 5' LCR of certain Alpha-9 HPVs

\begin{tabular}{llllll}
\hline HPV type & Start of LCR & Position of ORF & ORF length & Protein length $^{\text {MLOGD value }}$ \\
\hline HPV31 & 7073 & $7101-7394$ & $294 \mathrm{bp}$ & $97 \mathrm{aa}$ & 58.07 \\
HPV33 & 7094 & $7113-7463$ & $351 \mathrm{bp}$ & $116 \mathrm{aa}$ & 67.97 \\
HPV35 & 7110 & $7143-7316$ & $174 \mathrm{bp}$ & $57 \mathrm{aa}$ & 38.31 \\
HPV58 & 7140 & $7171-7353$ & $183 \mathrm{bp}$ & $60 \mathrm{aa}$ & 45.95 \\
\hline
\end{tabular}

${ }^{\text {a }}$ Total log likelihood ratio summed over phylogenetic tree. Positive values suggest that the tested ORFs are coding
ORFs were found in other HPV types belonging to the species Alpha-9 (HPV16, 52 and 67).

Next, the complete LCRs of these Alpha-9 HPVs (with ORFs in the 5' LCR) were obtained from PaVE (or GenBank in the case of HPV31) and aligned using the MACSE program [13]. The MACSE alignment tool was chosen because it aligns DNA sequences while considering their coding potential and provides codon-based alignments. The LCR alignment provided by MACSE was analysed by the MLOGD program to estimate the probability that these ORFs are protein-coding sequences (CDSs) [15]. The results of MLOGD analysis indicate that the ORF found in the 5' LCR of HPV33 is probably a protein-coding sequence (Supplementary Material 4). In addition, the ORFs found in the 5' LCRs of HPV31, 35 and 58 were also found to be CDSs with high probability as indicated by the high MLOGD values shown in Table 1.

Next, the coding sequences of the putative proteins potentially encoded by these $5^{\prime}$ LCR ORFs were obtained using ORF finder, aligned by MACSE and visualised by the SeaView software. As shown on Fig. 5, there is low similarity in the sequences of the putative proteins encoded by the 5' LCR ORFs of different Alpha-9 papillomaviruses. However, they have a very similar amino acid composition, with overrepresentation of cysteine and hydrophobic amino acids (especially valine and leucine) (Supplementary Material 5). Moreover, there are 5 cysteine residues at conserved positions in all of these putative proteins (in addition to 3 tyrosine, 2 valine and 1 leucine). Sequence similarity search of the putative proteins performed using Protein BLAST revealed no significant similarity to any known viral protein.

Protein structure prediction of the putative proteins encoded by these $5^{\prime}$ LCR ORFs (performed with the help of the QUARK Online tool) showed that they have a similar structure (Supplementary material 6). The putative proteins have 3-4 alpha helices (with loops connecting the helices) and they may have a membrane localisation (because of their highly hydrophobic nature). The putative protein potentially encoded by the HPV35 5' LCR ORF seems to have a somewhat different structure with 2 short alpha helices and 2 short beta sheets.

\section{Discussion}

The experimental data presented in this article indicate that the HPV33 LCR DNA sequence has an orientation-dependent toxic effect on the host bacteria that can be reduced by lowering the culture temperature to $25{ }^{\circ} \mathrm{C}$. This finding was quite unexpected as the LCR of genital HPVs is not known to code for any protein. In addition, cloning (and functional analysis) of the LCR of HPV33 or of closely related HPV types (HPV16 and 31) has been reported by several research groups, including the group of the authors [5-7, 20-22, 10]. The toxicity of an HPV LCR sequence on the host bacteria may depend on several factors like the presence or absence
Fig. 5 Amino acid sequence alignment of putative proteins potentially encoded by ORFs found in the 5' LCR of certain Alpha-9 HPVs. The putative coding sequences of ORFs shown in Table 1 were obtained from ORF finder and aligned by MACSE. The protein alignment produced by MACSE was visualised by the SeaView software. Conserved amino acids are shown in bold
1

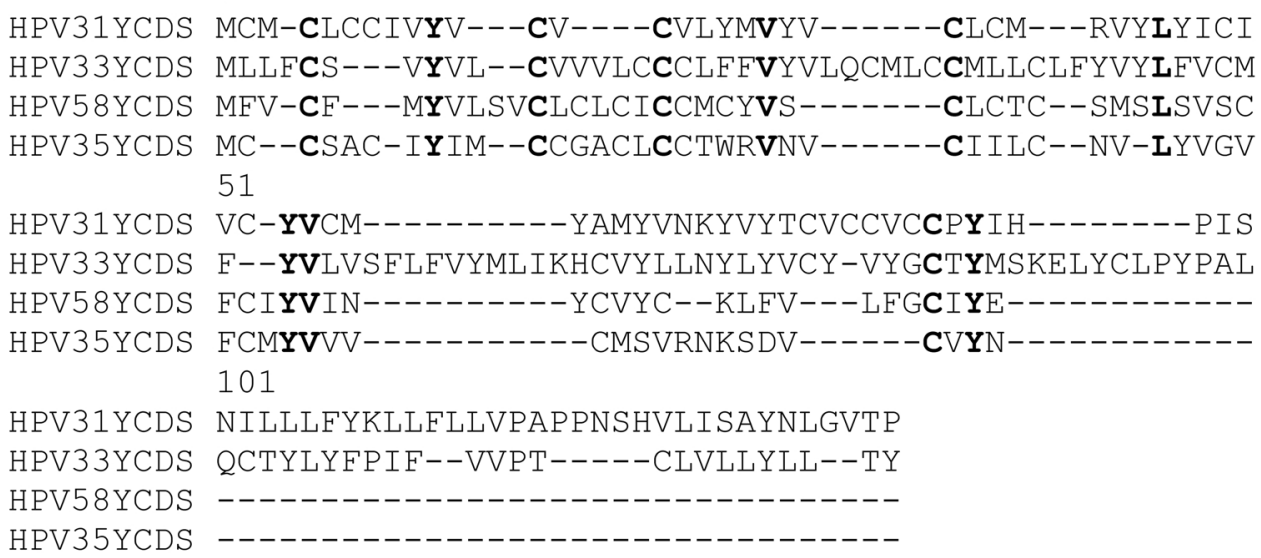


of an ORF in the LCR, the bacterial strain used for cloning, the cloning vector, and the segment of the LCR cloned (complete LCR or partial sequences). This might explain why this phenomenon has not been reported so far.

The temperature-dependent toxicity of HPV33 LCR in XL1 bacteria was confirmed by transformation experiments (Fig. 3). Not only the prototype HPV33 LCR sequence but also the natural sequence variants tested were found to have significant toxicity on the host bacteria at $37{ }^{\circ} \mathrm{C}$ but not at $25^{\circ} \mathrm{C}$ (Fig. 4a). The toxic effect of the LCR could be localised to the $5^{\prime}$ LCR segment (Figs. 1, 4b). As this part of the LCR was found to contain an ORF in HPV33 (Fig. 1 and Table 1), it was tempting to speculate that expression of a protein from this ORF may be responsible for the observed toxic effect on the host bacteria. Further reduction of the expression of this putative protein (by culturing at $25^{\circ} \mathrm{C}$ ) seems to attenuate the toxic activity of the protein.

Transformation experiments performed with mutant constructs indicated that disruption of the $5^{\prime}$ LCR ORF resulted in the reduction or the complete elimination of the toxic effect of the LCR on the host bacteria. Although the ORF encoding the 116-amino acid protein is disrupted in these constructs, some truncated ORFs are preserved potentially providing the expression of smaller proteins. This may be responsible for the remaining toxicity of the 33LCRD1mut1 construct on the host bacteria (Fig. 4b).

The putative product of the ORF found in HPV33 LCR may be a transmembrane protein (see later). The cloning of transmembrane proteins is known to be a challenge because of their toxicity [23, 24]. Therefore, even a low-level expression of this putative protein can be assumed to cause significant toxicity in the host bacteria. However, it is also possible that some other mechanism is responsible for the observed toxic effect of HPV33 LCR, such as the transcription of toxic non-coding RNA sequences. Therefore, further experiments should be performed to identify the exact mechanism that is responsible for the toxic effect of HPV33 LCR DNA on E. coli cells.

In silico analysis of the LCR of HPV33 and other HPV types belonging to the species Alpha-9 (HPV31, 35 and 58) seems to support the assumption that the ORFs found in the LCR of these HPVs are protein-coding sequences and may have some functional role in virus infected cells. These ORFs are present at similar locations in all of these four HPV types, between the L1 stop codon and the first E2 binding site (in the $5^{\prime}$ LCR segment). The ORF found in the 5' LCR of the HPV33 prototype was also conserved in each HPV33 LCR sequence variant analysed in this study (Supplementary material 3 ). The putative proteins that are supposed to be expressed from these ORFs have a similar amino acid composition (with high percentage of cysteine and hydrophobic amino acids). Although there is relatively low sequence similarity between the putative proteins of different HPV types, they could be aligned and the alignment revealed the presence of several conserved cysteine residues (Fig. 5). The conserved cysteines may be able to stabilise the structure of the proteins (by disulphide bonds) allowing relatively high sequence variation at other positions. This is a well-known characteristic of some cysteinerich proteins such as defensins [25].

To investigate further the possibility that the ORFs found in the 5' LCR of these Alpha-9 HPV types are protein-coding sequences, MLOGD analyses were performed using LCR alignment of these HPVs. The MLOGD software compares the probability of coding and non-coding (or single-coding and double-coding) models of a DNA sequence based on the nucleotide and amino acid changes found between the aligned sequences [15]. The results of MLOGD analysis seem to support the assumption that the ORFs found in the 5' LCR of these Alpha-9 HPV types are protein-coding sequences. Protein structure prediction suggested that these putative proteins have a predominantly alpha-helical structure and probably have a membrane localisation (suggested by the highly hydrophobic nature).

In conclusion, the results presented here indicate that the $5^{\prime}$ LCR segment of HPV33 has an orientation-dependent toxic effect on XL1 bacteria. The toxic effect is probably caused by the expression of a hydrophobic, cysteine-rich membrane protein from an ORF located in the 5' LCR. In silico analysis of this ORF and similar ORFs found in related HPV types (HPV31, 35 and 58) suggests that these ORFs may be protein-coding sequences. Further studies should be performed to prove that these putative proteins are really expressed in the infected host cells and to identify their function.

Acknowledgements Open access funding provided by University of Debrecen (DE). This study was supported by a grant from the Faculty of Medicine, University of Debrecen.

Author contributions The study was conceived and designed by GV. The experiments were performed by EG, AS and GV. Data analysis was performed by JK and GV. The first draft of the manuscript was written by GV. All authors read and approved the final manuscript.

\section{Compliance with ethical standards}

Conflict of interest The authors hereby declare that they have no competing interests.

Ethical approval Ethical approval for this study was obtained from the Regional Institutional Research Ethics Committee, University of Debrecen Clinical Center.

Open Access This article is licensed under a Creative Commons Attribution 4.0 International License, which permits use, sharing, adaptation, distribution and reproduction in any medium or format, as long as you give appropriate credit to the original author(s) and the source, provide a link to the Creative Commons licence, and indicate if changes were made. The images or other third party material in this article are 
included in the article's Creative Commons licence, unless indicated otherwise in a credit line to the material. If material is not included in the article's Creative Commons licence and your intended use is not permitted by statutory regulation or exceeds the permitted use, you will need to obtain permission directly from the copyright holder. To view a copy of this licence, visit http://creativecommons.org/licenses/by/4.0/.

\section{References}

1. Bernard HU, Burk RD, Chen Z, van Doorslaer K, Hausen H, de Villiers EM (2010) Classification of papillomaviruses (PVs) based on 189 PV types and proposal of taxonomic amendments. Virology 401:70-79

2. Doorbar J, Egawa N, Griffin H, Kranjec C, Murakami I (2015) Human papillomavirus molecular biology and disease association. Rev Med Virol 25(Suppl 1):2-23

3. Burk RD, Harari A, Chen Z (2013) Human papillomavirus genome variants. Virology 445:232-243

4. Bernard HU, Calleja-Macias IE, Dunn ST (2006) Genome variation of human papillomavirus types: phylogenetic and medical implications. Int J Cancer 118:1071-1076

5. Alvarez J, Gagnon D, Coutlee F, Archambault J (2018) Naturally occurring variations modulate the activity of the HPV33 early promoter and its affinity for the E2 transcription factor. Sci Rep $8: 15015$

6. Ferenczi A, Gyöngyösi E, Szalmás A, Hernádi Z, Tóth Z, Kónya J, Veress G (2013) Sequence variation of human papillomavirus type 31 long control region: phylogenetic and functional implications. J Med Virol 85:852-859

7. Kammer C, Warthorst U, Torrez-Martinez N, Wheeler CM, Pfister $H$ (2000) Sequence analysis of the long control region of human papillomavirus type 16 variants and functional consequences for P97 promoter activity. J Gen Virol 81:1975-1981

8. Lopez-Saavedra A, Gonzalez-Maya L, Ponce-de-Leon S, GarciaCarranca A, Mohar A, Lizano M (2009) Functional implication of sequence variation in the long control region and E2 gene among human papillomavirus type 18 variants. Arch Virol 154:747-754

9. Sichero L, Franco EL, Villa LL (2005) Different P105 promoter activities among natural variants of human papillomavirus type 18. J Infect Dis 191:739-742

10. Veress G, Szarka K, Dong XP, Gergely L, Pfister H (1999) Functional significance of sequence variation in the $\mathrm{E} 2$ gene and the long control region of human papillomavirus type 16. J Gen Virol 80(Pt 4):1035-1043

11. Bernard HU (2013) Regulatory elements in the viral genome. Virology 445:197-204

12. Geissmann Q (2013) OpenCFU, a new free and open-source software to count cell colonies and other circular objects. PLOS ONE $8: \mathrm{e} 54072$
13. Ranwez V, Harispe S, Delsuc F, Douzery EJ (2011) MACSE: multiple alignment of coding SEquences accounting for frameshifts and stop codons. PLoS ONE 6:e22594

14. Firth AE, Brown CM (2005) Detecting overlapping coding sequences with pairwise alignments. Bioinformatics 21:282-292

15. Firth AE, Brown CM (2006) Detecting overlapping coding sequences in virus genomes. BMC Bioinform 7:75

16. Xu D, Zhang Y (2012) Ab initio protein structure assembly using continuous structure fragments and optimized knowledge-based force field. Proteins 80:1715-1735

17. Xu D, Zhang Y (2013) Toward optimal fragment generations for ab initio protein structure assembly. Proteins 81:229-239

18. Gouy M, Guindon S, Gascuel O (2010) SeaView version 4: A multiplatform graphical user interface for sequence alignment and phylogenetic tree building. Mol Biol Evol 27:221-224

19. Zheng X, Tong W, Liu F, Liang C, Gao F, Li G, Tong G, Zheng $\mathrm{H}$ (2016) Genetic instability of Japanese encephalitis virus cDNA clones propagated in Escherichia coli. Virus Genes 52:195-203

20. Kim YB, Song YS, Jeon YT, Park JS, Um SJ, Kim JW, Park NH, Kang SB, Lee HP (2005) Sequence variation and the transcriptional activity of the upstream regulatory region in human papillomavirus 16 E7 variants in cervical cancer of Korean women. Oncol Rep 14:459-464

21. Park JS, Hwang ES, Lee CJ, Kim CJ, Rha JG, Kim SJ, Namkoong SE, Um SJ (1999) Mutational and functional analysis of HPV16 URR derived from Korean cervical neoplasia. Gynecol Oncol 74:23-29

22. Veress G, Murvai M, Szarka K, Juhász A, Kónya J, Gergely L (2001) Transcriptional activity of human papillomavirus type 16 variants having deletions in the long control region. Eur J Cancer 37:1946-1952

23. Miroux B, Walker JE (1996) Over-production of proteins in Escherichia coli: mutant hosts that allow synthesis of some membrane proteins and globular proteins at high levels. J Mol Biol 260:289-298

24. Montigny C, Penin F, Lethias C, Falson P (2004) Overcoming the toxicity of membrane peptide expression in bacteria by upstream insertion of Asp-Pro sequence. Biochim Biophys Acta 1660:53-65

25. Shafee TM, Robinson AJ, van der Weerden N, Anderson MA (2016) Structural homology guided alignment of cysteine rich proteins. Springerplus 5:27

Publisher's Note Springer Nature remains neutral with regard to jurisdictional claims in published maps and institutional affiliations. 